\title{
L'erogazione di prestazioni sanitarie in telemedicina
}

\author{
Francesco Burrai ${ }^{1}$, Margherita Gambella ${ }^{2}$, Angelica Scarpa ${ }^{2}$ \\ ${ }^{1}$ SC Formazione, Ricerca e Cambiamento Organizzativo, ATS Sardegna - ASSL di Sassari, Sassari - Italy \\ ${ }^{2}$ Facoltà di Medicina e Chirurgia, Università degli Studi di Sassari, Sassari - Italy
}

\begin{abstract}
Providing healthcare services in telemedicine
As a consequence of the Sars-CoV2 pandemic, the role of telemedicine in the healthcare sector was reviewed in order to ensure continuity of care and to contain infections. This new frontier involves professionals, caregivers, patients and can be applied in various fields, such as surgery, specialist visits, follow-ups, home care, health education, prevention and monitoring. Despite privacy may seem a deterrent for the implementation of this new goal in the healthcare sector, the community and the national legislation both provide rules to protect individuals and the community. This innovative method of assistance brings with it limits that must not detract attention from the great possibilities arising from the use of telemedicine.
\end{abstract}

Keywords: E-health, Mobile health, Telehealth, Telemedicine, Privacy

\section{Introduzione}

Con l'avanzare della tecnologia, si è reso necessario stare al passo con i tempi e adattare i modelli sanitari tradizionali alle innovazioni tecnologiche, tra cui le tecnologie mobili per supportare la sanità, definite mobile health (mHealth) e telehealth o telemedicina (TM), sono quelle fondamentali (1).

In questo particolare periodo storico segnato dalla pandemia da Covid-19, è evidente la necessità di una sanità non più convenzionale, potremmo dire analogica, ma digitale, la $T M$, che garantisca la continuità assistenziale e la qualità dell'assistenza in sinergia con le prestazioni svolte in presenza (2). L'obiettivo della TM non è quello di sostituire i sistemi sanitari tradizionali, bensì di supportarli e di implementarli favorendo lo scambio di informazioni. Anche l'Organizzazione Mondiale della Sanità (OMS) si è espressa in merito alla TM, pubblicando delle Linee Guida con lo scopo di fornire raccomandazioni per gli interventi di salute digitale, come visite specialistiche, assistenza domiciliare dei pazienti con malattie croniche ed educazione sanitaria (3). Se il punto di forza della TM consiste nell'abbattere le distanze e nel permettere

Received: January 1, 2021

Accepted: January 18, 2021

Published online: February 18, 2021

Indirizzo per la corrispondenza:

Francesco Burrai

Complesso Sanitario San Camillo

Strada Statale 200

07100 Sassari - Italy

francescoburrai@libero.it un rapido e tracciato accesso alle cure, d'altra parte condivide con il sistema sanitario problematiche comuni alle prestazioni in presenza, come inefficienze gestionali e una cultura dei sanitari e della popolazione ancora insufficiente (3). Nel panorama italiano, il Ministero della Salute ha prodotto un fondamentale documento con le indicazioni nazionali e i campi di applicazione in riferimento all'erogazione delle prestazioni in TM evidence-based (2).

\section{Campi di applicazione in telemedicina}

Uno degli aspetti più importanti della TM è la condivisione di informazioni sanitarie tra equipe interdisciplinari nazionali e internazionali. La condivisione di informazioni cliniche tramite la TM può avvenire tra sanitari, tra sanitari e pazienti e tra sanitari e caregiver. La comunicazione tra sanitari in TM può portare notevoli vantaggi nel campo dell'emergenzaurgenza ed educativo. Uno studio di Gulacti e Lok del 2017 affronta il drammatico problema dell'affollamento dei Pronto Soccorso (PS), che risulta essere associato a una permanenza prolungata nel PS dovuta a ritardi delle visite mediche da parte del personale medico specialista. In questo contesto assistenziale è stata utilizzata un'applicazione di messaggistica sicura per la condivisione di immagini, video, file audio, messaggi e documenti di carattere sanitario. I risultati hanno mostrato che ben il $61,8 \%$ delle consultazioni è stato completato utilizzando solo l'applicazione di messaggistica sicura, senza la presenza fisica del medico specialista in PS. Lo studio ha dimostrato che questo metodo riduce il tempo di consultazione e l'affollamento in PS rispetto alla consultazione telefonica tradizionale (4). Dal punto di vista dell'educazione, la mHealth rappresenta un metodo alternativo 
di apprendimento sia per i futuri sanitari sia per quelli già formati. L'obiettivo strategico è quello di inserire immediatamente i futuri professionisti nelle diverse tecnologie, dunque inserendo nel loro background le nuove competenze digitali applicate alla sanità, e di introdurre i professionisti già formati verso una nuova realtà sanitaria (5).

I benefeci della comunicazione a distanza comprendono anche la riduzione della lontananza tra sanitari e pazienti (6). Per esempio, nei contesti extraurbani, molto spesso sono presenti problematiche di carenze di personale sanitario sul territorio (7) e la presenza, invece, della TM con la sua comunicazione a distanza può ridurre questo divario fisico e comunicativo. La comunicazione tra sanitari e pazienti può essere utile anche per l'educazione, la promozione della salute e la gestione del follow-up (8), ma anche per una maggiore aderenza alla terapia (3). Lo studio condotto da Wang et al. del 2019 evidenzia come l'utilizzo di un'applicazione mobile per la gestione del diabete impatti positivamente e a lungo termine sui livelli di emoglobina glicata (HbA1c) nei pazienti affetti da diabete mellito di tipo 1 (9).

La telechirurgia è un'altra branca della TM e consente ai chirurghi di operare in remoto pazienti che si trovano in sale operatorie distanti dal chirurgo e questo è permesso da reti wireless e dalla tecnologia robotica. Oltre a eliminare le barriere fisiche e geografiche, la telechirurgia è una chirurgia generalmente di alta qualità, eseguita da specialisti di elevate capacità chirurgiche e che permette, sul versante economico, anche un abbattimento dei costi per l'utenza (10), che non deve spostarsi in altre regioni o in altre nazioni. Un altro campo della telechirurgia è quello urologico, in cui questo nuovo strumento digitale è stato oggetto di studio per la gestione di pazienti con prolasso degli organi pelvici, infezioni urinarie e calcoli urinari (11).

Un'altra popolazione che ha beneficiato della TM è quella dei caregiver, degli assistenti e/o dei familiari che prestano assistenza al paziente. Una revisione sistematica sulla TM come supporto ai caregiver suggerisce che l'uso dei servizi sanitari a distanza fornisce un monitoraggio efficiente per i malati cronici al pari delle cure tradizionali e impatta positivamente sulla qualità di vita dei caregiver stessi. Ciò consente loro di risparmiare sui costi degli spostamenti verso le strutture sanitarie, migliorando anche la loro salute psicologica (12).

\section{Privacy e consenso informato}

Il tema della riservatezza dei dati personali e della privacy è un tema molto delicato e sentito dalla collettività. A livello europeo, queste tematiche sono disciplinate dal Regolamento UE 2016/679, GDPR (13). Un problema persistente nell'utilizzo di device digitali collegati a una rete e, dunque, nel trasferimento e nella condivisione di dati personali e sanitari in sicurezza è rappresentato da possibili attacchi da parte di hacker e, di conseguenza, da una persistente problematica di cybersecurity. A livello Italiano le Linee Guida del Ministero della
Salute indicano i requisiti essenziali per la salvaguardia dei dati personali tramite sistemi di gestione della cybersecurity (2).

Uno strumento strategico per la consultazione dei dati sanitari è il fascicolo sanitario elettronico (FSE). II FSE è I'insieme dei dati e documenti digitali di tipo sanitario e sociosanitario generati da eventi clinici presenti e trascorsi riguardanti l'assistito, riferiti anche alle prestazioni erogate al di fuori del Servizio Sanitario Nazionale (14).

La TM presenta due classici aspetti critici, che sono il consenso informato del paziente e la responsabilità sanitaria. II primo garantisce la trasparenza delle prestazioni in TM erogate all'utente, il quale deve conoscere il tipo di trattamento, gli obiettivi e le modalità di gestione dei propri dati sensibili (2). II secondo regolamenta le responsabilità del professionista della salute verso l'utente durante le fasi assistenziali in TM. I fattori come la presa in carico dell'utente e il rispetto delle norme legislative, deontologiche e bioetiche devono essere applicati e rispettati come nelle prestazioni sanitarie tradizionali (2). Ogni azione sanitaria in TM deve essere coerente con l'articolo 32 della Costituzione Italiana (15).

\section{Elementi necessari per le prestazioni sanitarie in telemedicina}

Per poter usufruire del servizio di TM è necessario che l'utente abbia dei requisiti hardware, software e di conoscenza informatica minimi. La prestazione sanitaria in TM può essere effettuata se l'utente possiede un contatto telematico, strumento per poter comunicare tramite specifici device con i sanitari. In caso l'utente non disponga di dispositivi quali telefono cellulare, tablet o computer collegati a una rete Internet, non verrà comunque escluso dal servizio, bensì gli verrà garantita la possibilità di accedere ai servizi per l'erogazione della prestazione, ma non, ovviamente, in TM (2). Così come per gli utenti, anche ai sanitari è richiesta la presenza di un collegamento a Internet e di un portale web per la gestione delle televisite, compatibilmente con le norme vigenti sulla privacy e sulla sicurezza (2).

\section{Limiti di applicazione}

L'utilizzo di queste tecnologie necessita obbligatoriamente di una connessione Internet, di una conoscenza informatica e della disponibilità di device. La mancanza di questi requisiti, data, molto spesso, da un'età avanzata, da una situazione socioeconomica critica o da una posizione geografica sfavorevole per la connessione Internet, può rappresentare un ostacolo all'applicazione della TM.

Ancora oggi, un fattore limitante è dato dalla non disponibilità in tutti gli ospedali e ambulatori di accesso agli strumenti di TM (16) oppure dall'elevato costo dei software e delle fotocamere digitali (17). Un altrettanto importante fattore limitante è il gap delle conoscenze informatiche da parte dei sanitari con, di conseguenza, la necessità di costruire un 
percorso formativo specifico per acquisire le competenze necessarie all'utilizzo dei nuovi strumenti digitali applicati alla sanità (17).

Nello studio qualitativo di Odendaal et al. del 2020, la TM mostrava alcuni limiti nella sua applicazione, dovuti alla percezione, da parte degli utenti, di non poter avere una relazione fisica, faccia a faccia con i sanitari, oppure diversi utenti mostravano scetticismo nell'efficacia della TM. Lo studio mostrava anche come i sanitari con meno esperienza evidenziavano una maggiore preoccupazione nel caso di errori commessi di fronte agli utenti, mentre altri sanitari hanno sentito la loro capacità clinica minacciata (18).

Infine, la TM non è uno strumento assistenziale consigliato quando i pazienti presentano patologie acute o patologie croniche associate a fragilità o disabilità, perché la permanenza a domicilio per questo tipo di assistiti potrebbe essere un'imprudenza (2).

\section{Conclusioni}

La TM consente l'accesso alle cure anche a quei soggetti che si trovano fisicamente lontani e impossibilitati a raggiungere la struttura sanitaria o un ambulatorio. La TM rende immateriale la struttura sanitaria e raggiunge il paziente eliminando i confini fisici.

La TM, con il suo sistema di tracciamento e di memorizzazione delle informazioni, consente di risolvere problemi come l'assenza di dati per perdita di dati registrati su supporti cartacei o incomprensibilità di alcune scritture nei supporti cartacei. Tali problemi che banali non sono e che, purtroppo, sono ancora presenti in molte realtà sanitarie possono impattare negativamente nei percorsi di follow-up oppure quando un paziente si sposta da una struttura sanitaria a un'altra, con casi non rari in cui si deve ricostruire nuovamente un'anamnesi medica, con notevole perdita di tempo e di informazioni.

La TM permette la condivisione sicura dei dati tramite sistemi criptati e garantisce una migliore continuità assistenziale, innescando una maggiore fiducia da parte del cittadino verso i sanitari e la sanità in generale. Un esempio frequente potrebbe essere quello dei pazienti non autosufficienti o anziani, criticità aggravata in questo periodo di pandemia da Covid-19, i quali si trovano soli al momento del ricovero e non riescono a comunicare informazioni riguardanti la loro situazione di salute attuale e pregressa, comprese eventuali allergie e terapie farmacologiche in corso. Con I'utilizzo della TM nella quotidianità assistenziale, anche questo disagio potrebbe essere risolto.

In questo periodo di pandemia da Covid-19, in cui la sanità ha dovuto "chiudere le porte" ai caregiver, questi ultimi hanno dovuto utilizzare in maniera frequente le chiamate telefoniche per la trasmissione di informazioni sensibili riguardanti i pazienti. Appare chiaro che questa modalità di trasmissione dei dati può andare incontro a perdita di informazioni o a errori da parte dei sanitari nella trascrizione dei dati.
Anche nelle situazioni di emergenza o durante i trasferimenti dei pazienti nei reparti di degenza, una visione tempestiva dei dati sanitari attraverso una cartella telematica e l'acquisizione veloce e precisa dei dati dell'utente possono accelerare la presa in carico da parte dei sanitari, i quali potranno essere maggiormente preparati nell'accoglierlo nell'unità operativa e nell'assisterlo tramite un piano di assistenza individuale. Uno studio condotto da Powell et al. del 2017 sottolinea la positività dell'esperienza dell'assistenza a distanza tramite la TM, la quale ha dato la possibilità a diversi utenti di non perdere una giornata lavorativa e, ad altri, la sensazione confortevole di trovarsi nelle proprie mura domestiche, migliorando la compliance del paziente e, così, l'assistenza stessa (19).

La realtà sanitaria e sociosanitaria contemporanea in Italia impone la TM. La TM può essere uno strumento, se ben organizzata e gestita, capace di migliorare l'efficacia, l'efficienza e l'appropriatezza delle prestazioni sanitarie e sociosanitarie. Appare anche evidente che le nuove generazioni, le generazioni dei Millennial che vivono le tecnologie digitali e la connessione come fatti ineludibili nel loro quotidiano, fanno sperare in un futuro in cui la TM sarà alla portata di tutti, indistintamente dalla provenienza geografica, dal ceto socioculturale e dalle disponibilità economiche.

\section{Disclosures}

Conflict of interest: The authors declare no conflict of interest. Financial support: This research received no specific grant from any funding agency in the public, commercial, or not-for-profit sectors.

\section{Bibliografia}

1. WHO 2011. mHealth: New horizons for health through mobile technologies: second global survey on eHealth. Online (data ultimo accesso 30/12/2020).

2. Art. 4, comma 1, del decreto legge 28 agosto 1997, n. 281 "Indicazioni nazionali per l'erogazione di prestazioni in telemedicina". Online (data ultimo accesso 30/12/2020).

3. WHO 2019. World Health Organization. WHO Guideline: Recommendations on Digital interventions for Health System Strengthening. Online (data ultimo accesso 28/12/2020).

4. Gulacti U, Lok U. Comparison of secure messaging application (WhatsApp) and standard telephone usage for consultations on Length of Stay in the ED. A prospective randomized controlled study. Appl Clin Inform. 2017;8(3):742-753. CrossRef PubMed

5. Jumreornvong $O$, Yang E, Race J, Appel J. Telemedicine and Medical Education in the Age of COVID-19. Acad Med. 2020;95(12):1838-1843. CrossRef PubMed

6. Telemedicine opportunities and developments in Member States: report on the second global survey on eHealth. Geneva: World Health Organization; 2010. Global Observatory for eHealth series, volume 2. Online (data ultimo accesso 30/12/2020).

7. World Health Organization. Increasing access to health workers in remote and rural areas through improved retention: global policy recommendations. Geneva: World Health Organization; 2010. Online (data ultimo accesso 30/12/2020).

8. Classification of digital health interventions v1.0: a shared language to describe the uses of digital technology for health. 
Geneva: World Health Organization; 2018. WHO/RHR/18.06. Online (data ultimo accesso 30/12/2020).

9. Wang $X$, Shu W, Du J, et al. Mobile health in the management of type 1 diabetes: a systematic review and meta-analysis. BMC Endocr Disord. 2019;19(1):21. CrossRef PubMed

10. Choi PJ, Oskouian RJ, Tubbs RS. Telesurgery: Past, present, and future. Cureus. 2018 maggio 31;10(5):e2716. CrossRef

11. Novara G, Checcucci E, Crestani A, et al. Research Urology Network (RUN). Telehealth in Urology: A Systematic Review of the Literature. How Much Can Telemedicine Be Useful During and After the COVID-19 Pandemic? Eur Urol. 2020;78(6): 786-811. CrossRef PubMed

12. Chi N, Demiris G. A systematic review of telehealth tools and interventions to support family caregivers. J Telemed Telecare. 2015 gennaio;21(1):37-44. CrossRef

13. REGOLAMENTO (UE) 2016/679 del Parlamento Europeo e del Consiglio del 27 aprile 2016. Online (data ultimo accesso 30/12/2020).
14. Artt. 12 e 13, comma 1 , del decreto legge 18 ottobre 2012, n. 179. Online (data ultimo accesso 30/12/2020).

15. La Costituzione Italiana, Parte I, Titolo II, articolo 32. Online (data ultimo accesso 30/12/2020).

16. Asiri A, AlBishi S, AlMadani W, ElMetwally A, Househ M. The Use of Telemedicine in Surgical Care: a Systematic Review. Acta Inform Med. 2018;26(3):201-206. CrossRef PubMed

17. Robie DK, Naulty CM, Parry RL, et al. Early experience using telemedicine for neonatal surgical consultations. J Pediatr Surg. 1998;33(7):1172-1176. CrossRef PubMed

18. Odendaal WA, Anstey Watkins J, Leon N, et al. Health workers' perceptions and experiences of using mHealth technologies to deliver primary healthcare services: a qualitative evidence synthesis. Cochrane Database Syst Rev. 2020;3(3):CD011942. CrossRef PubMed

19. Powell RE, Henstenburg JM, Cooper G, Hollander JE, Rising KL. Patient Perceptions of Telehealth Primary Care Video Visits. Ann Fam Med. 2017;15(3):225-229. CrossRef PubMed 